\title{
On the spatial linear growth of gravity-capillary water waves sheared by a laminar air flow
}

\author{
Y. S. Tsai \\ J. M. Burgers Centre for Fluid Dynamics, Laboratory for Aero- and Hydrodynamics, \\ Delft University of Technology, Leeghwaterstraat 21, 2628 CA Delft, The Netherlands \\ A. J. Grass and R. R. Simons \\ Department of Civil and Environmental Engineering, University College London, Gower Street, \\ London WC1E 6BT, United Kingdom
}

(Received 6 December 2004; accepted 14 July 2005; published online 30 August 2005)

\begin{abstract}
The initial growth of mechanically generated small amplitude water waves below a laminar air stream was examined numerically and experimentally in order to explore the primary growth mechanism, that is, the interfacial instability of coupled laminar air and water flows. Measurements of the laminar velocity profile in the air over the water surface were found to be consistent with Lock's [Q. J. Mech. Appl. Math. 4, 42 (1951)] theory. This profile was then used to calculate the spatial growth rates by solving the Orr-Sommerfeld equations. The simulation shows that the growth of the boundary layer affects the exponential growth of water waves along the fetch. The sensitivity of the growth rate is observed to vary by a factor of 2 for changes in the laminar velocity profile as small as $2 \%$ at the water surface. This indicates that the interfacial instability is strongly influenced by the wind-induced surface current. A laminar airflow was produced in the wind tunnel over mechanically generated monochromatic gravity-capillary water waves with the $k a$ value in the order of $10^{-3}$. The novel experiment was designed to measure the minute changes in the wave slope and phase velocity simultaneously using a highly sensitive reflected twin laser beam technique. Agreement between linear theory and experiments for the spatial development of wave height and phase velocity suggests that the linear instability mechanism determines the initial stages of development of small-scale water waves. () 2005 American Institute of Physics.
\end{abstract}

[DOI: $10.1063 / 1.2033910]$

\section{INTRODUCTION}

The formation of interfacial disturbances caused by shear flow instability occurs in a wide range of flow situations of engineering interest including, for example, a thinfilm flow of liquid over a solid surface, air-sea interaction, the mixing process in a jet or wake, and fluid flow over a compliant surface. One of the most familiar phenomena in nature, which has attracted considerable interest and study over the past century, is that of the generation of water waves by wind. Different mechanisms have been proposed in attempting to explain the central problem of energy transfer from the wind to the waves. These models are only relevant under particular conditions and at different stages of wave growth and wave development. A definitive explanation of the wave growth mechanism either physical or theoretical remains to be propounded at the present time.

In his pioneering study, Miles ${ }^{1}$ considered how water waves grow when the mean wind velocity has a linearlogarithmic profile assuming the distribution in the airflow is governed by the inviscid Rayleigh equation. This so-called quasilaminar model resulted in the prediction of a constant exponential growth rate. For a small-scale wave, Benjamin ${ }^{2}$ contributed a major advancement to the understanding of the role of viscosity in the growth of waves on a general flexible surface through a wall frictional layer adjacent to the boundary. The energy transferring from wind to waves was ex- plained by the distribution of shear and normal stresses over a wavy boundary. Miles ${ }^{3}$ generalized the previous work of Benjamin and, ignoring the wind-induced surface current, demonstrated that the resonance between the TollmienSchlichting waves and free-surface waves provides the mechanism for water wave growth. Subsequent studies incorporated some minor but also important additional factors such as surface current ${ }^{4,5}$ and distributions of the velocity profiles. ${ }^{5,6}$ These studies focused mainly on the application of a turbulent shear layer over a wavy surface in order to simulate the natural state of water wave growth. Comparison with the laboratory experiments of Larson and Wright ${ }^{7}$ and Kawai ${ }^{8}$ indicated a high probability that the wave growth can be predicted by linear instability theory in the initial stages.

From the models mentioned above, it has been concluded that the precise form of the wind shear profile is critical to the nature of the instability of the water waves. However, a turbulent flow developing over a deformed water surface is a highly complex phenomenon in which the role of the air-water interface is analogous to that of a solid surface. Streaky structures and bursting near the interface and largescale ordered motion in the outer part of the boundary layer violently perturbing the entire boundary shear flow have been observed. ${ }^{9,10}$ At present, this knowledge can only come from direct numerical simulation and advanced physical experimentation, paralleling similar studies of solid wall layer turbulence. ${ }^{11,12}$ The complexity of the interaction process 
leads to uncertainties in the determination of the friction velocity on an undulating surface and even the exact shape of the turbulent velocity profile. These are, however, precisely the fundamental factors responsible for the probability of significant discrepancies in the wave growth rate prediction from linear instability theory.

Originating from the analysis of the instability of a laminar flow over a rigid surface, under the assumption of infinitely small disturbances superimposed on the mean flow, linear instability theory has been used to describe the development of Tollmien-Schlichting waves and the theory has been well confirmed. ${ }^{13-15}$ The difficulty in using a turbulent airflow over a heavy liquid is that significant oscillations of the primary flow especially near the boundary caused by turbulent inrushes and ejections contravene the primary assumption of small disturbance theory. As has also been pointed out by Benjamin ${ }^{16}$ this linearized theory only applies to laminar shear flows. In this respect Miles' model and those of later researchers who also used a mean velocity distribution to represent a turbulent flow remain questionable. Consequently, in discussing the formation of water waves, use of the Orr-Sommerfeld equation may only be appropriate for cases with laminar boundary layers on both sides of the interface.

Blennerhassett ${ }^{17}$ and Blennerhassett and Smith ${ }^{18}$ developed a laminar model to study the stability of the air/water interface using a Poiseuille-Coutte-type velocity profile. The model shows that surface waves can be generated by a laminar model for a range of different wavelengths. The velocity profile for general two layer laminar streams on the interface was analyzed by Lock. ${ }^{19}$ For the case of airflow over a water surface, referred to here as the "Lock profile," the profile was closely similar to the Blasius profile with a difference of approximately $2 \%$. Lock ${ }^{20}$ examined the air-water interfacial stability using the Lock profile. The curves for neutral stability of the two distinct types of waves, "water waves" and "air waves," were explored and the generation of water waves by wind was discussed in relation to these neutral curves. The Lock profile was used by Akylas ${ }^{21}$ to build a model of the resonance mechanism proposed by Miles. ${ }^{3}$ The mechanism was found to dominate the wave growth for wavelengths greater than $12 \mathrm{~cm}$. The use of the Lock profile in the literature of wind-wave interaction can be found only in the above two studies. This may be because there was no experimental evidence to validate Lock's theory. In a recent wind tunnel study, Tsai ${ }^{22}$ and Tsai et al. ${ }^{23}$ have accurately measured the profile over the moving water surface.

Gupta et al. ${ }^{24}$ reported the first and only known experiment to look specifically at the growth of free-surface waves under the action of a laminar air stream for the original purpose of using water as a compliant material to study drag reduction. However, their measurements were not in good agreement with the theoretical prediction with respect either to the laminar velocity profile shape or to the surface water wave growth. The experimental equipment used probably restricted the investigation.

In the present study both experimental and numerical simulations have been carried out in an attempt to improve the understanding of the growth process of water waves driven by the Lock boundary layer. Novel techniques have been developed to make an experimental investigation possible and to facilitate corroboration of the experiments using the numerical simulation. It is important to note that direct comparisons between the prediction and measurements of the wave growth with respect to distance do not appear to have been made previously. The problem under consideration and the structure of this paper are as follows. Section II discusses the relevant theories including wave attenuation in a water tank and linear instability theory to predict the spatial growth of short water waves. Section III outlines the numerical procedure and presents the simulation results. Section IV describes the experimental setup used to generate and measure small long-crested surface waves. Section V presents the results of these measurements in comparison with the numerical simulations. Related discussion and conclusions are presented in Secs. VI and VII.

\section{THEORY}

\section{A. Wave attenuation}

For small-scale waves, the surface-tension force increases in significance relative to gravity as a restoring force. A gravity-capillary water wave is said to exist in the range where the gravity force is dominant and the surface-tension force cannot be ignored. This range is often expressed in terms of the Bond number

$$
B=\frac{\rho_{w}^{-1} T k^{2}}{g},
$$

which expresses the relative strength of the surface tension. Here, $\rho_{w}$ is the density of water, $T$ is surface tension, $k$ is the wave number, and $g$ is gravity. Water waves with frequencies between $4 \mathrm{~Hz}(B \approx 0.03)$ and $13.45 \mathrm{~Hz}(B=1$, which marks the occurrence of the minimum phase velocity, taking $T$ $=0.074 \mathrm{~N} \mathrm{~m}^{-1}, \quad w=999.5 \mathrm{~kg} \mathrm{~m}^{-3}$ at $12{ }^{\circ} \mathrm{C}, \quad$ and $g$ $=9.81 \mathrm{~ms}^{-2}$ ) are considered gravity-capillary waves.

When these small-scale water waves propagate downstream in a wave flume, the wave height decays significantly due to internal and boundary energy dissipation arising from viscous stress and bottom and sidewall frictions, respectively. Lamb ${ }^{25}$ showed that the temporal damping of wave height follows an exponential decay of the form

$$
H=H_{o} \exp \left(-\zeta_{t} t\right),
$$

where $H_{o}$ is the initial wave height, $\zeta_{t}$ is the temporal damping rate, which is the linear superposition of viscous damping rate, $\zeta_{\nu}$, and boundary friction, $\zeta_{b}$,

$$
\zeta_{t}=\zeta_{\nu}+\zeta_{b} .
$$

The surface damping coefficient due to viscosity is

$$
\zeta_{\nu}=2 \nu_{w} k^{2},
$$

where $\nu_{w}$ is the kinematic water viscosity. The boundary damping coefficient has a contribution from friction at the bottom and the side walls, ${ }^{26,27}$ 


$$
\zeta_{b}=\sigma\left(\frac{\nu_{w}}{2 \sigma}\right)^{1 / 2} \frac{k}{\sinh 2 k h}+\frac{\sigma}{b}\left(\frac{\nu_{w}}{2 \sigma}\right)^{1 / 2},
$$

where $\sigma$ is angular frequency and $b$ is the width of the flume.

The temporal damping can be transformed to spatial damping through Gaster's relation, ${ }^{28}$

$$
\zeta_{x}=\zeta_{t} / c_{g},
$$

where $c_{g}$ is the group velocity. In deep water, the bottom dissipation is negligible and the damping rate with respect to distance becomes

$$
\zeta_{x}=\left[2 \nu_{w} k^{2}+\frac{\sigma}{b}\left(\frac{\nu_{w}}{2 \sigma}\right)^{1 / 2}\right] / c_{g} .
$$

\section{B. Unperturbed boundary layer at the air-water interface}

Lock $^{19}$ developed the general case for two immiscible viscous fluid flows with different densities, viscosities, and velocities shearing along an interface and hence forming a mixing layer in both fluids. In the present study, the theory is applied to a uniform laminar airflow, occupying the region $y^{*}>0$, shearing over an infinite quiescent water basin. The contact point between the two fluids occurs at $x^{*}=0$, where $x^{*}$ and $y^{*}$ represent the Cartesian coordinates in the horizontal and vertical directions, respectively. $y^{*}$ is measured upwards from the horizontal interface. The flow system forms two Blasius equations to describe the upper and lower flows, respectively,

$$
\begin{aligned}
& f_{a} f_{a}^{\prime \prime}+2 f_{a}^{\prime \prime \prime}=0, \\
& f_{w} f_{w}^{\prime \prime}+2 f_{w}^{\prime \prime \prime}=0,
\end{aligned}
$$

through the use of a similarity variable $\eta=y^{*} \sqrt{U_{a_{\infty}} / \nu x^{*}}$ and a stream function of the form $\psi=\sqrt{\nu x^{*} U_{a_{x}}} f(\eta)$, where subscripts $a$ and $w$ denote the air and water, $U_{a_{\infty}}$ is the air freestream velocity, $\nu$ is the kinematic viscosity, and the primes denote the derivatives with respect to $\eta$.

The boundary conditions at the plane of interface are continuity of stream function and velocity, and balance of shear stress:

$$
\begin{aligned}
& \eta_{a}=\eta_{w}=0: f_{a}=f_{w}=0, \\
& \eta_{a}=\eta_{w}=0: f_{a}^{\prime}=f_{w}^{\prime}, \\
& \eta_{a}=\eta_{w}=0: \rho_{a} \sqrt{\nu_{a}} f_{a}^{\prime \prime}=\rho_{w} \sqrt{\nu_{w}} f_{w}^{\prime \prime},
\end{aligned}
$$

and the boundary conditions at infinity are

$$
\eta_{a} \rightarrow+\infty: f_{a}^{\prime}=1, \quad \eta_{w} \rightarrow-\infty: f_{w}^{\prime}=0 .
$$

The exact solution of the boundary layer profile is obtained from the integration of the Blasius equations (8) and (9) as plotted in Fig. 1 in comparison with the Blasius profile. For the Lock profile, a slip condition on the interface means that a weak surface current is induced by the wind. The surface flow is $2.13 \%$ of the free-stream velocity. The Lock velocity profile is similar to that of the Blasius profile with a difference generally less than $2 \%$ because the ratio of
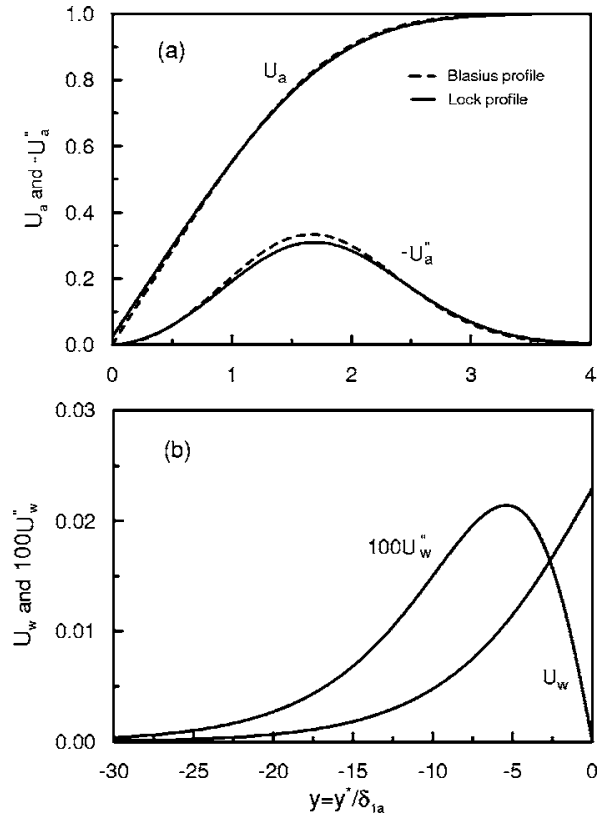

FIG. 1. (a) Lock and Blasius profiles and their curvature in the air. (b) Lock profile and its curvature in the water. $\delta_{1 a}$, boundary layer displacement thickness in the air.

air to water density is small. The negative curvature in the air velocity profile is essential in linear instability theory to excite unstable modes. Although the maximum difference between the two curvatures of the two profiles is less than 7\%, it causes a disproportionate difference to the wave growth rate. This point will be discussed in Sec. III C.

Figure 2 demonstrates the measurements for the Lock profile in air. ${ }^{22,23}$ The agreement between the experiment and theory validates the profile adopted in the present model to drive the Orr-Sommerfeld equation.

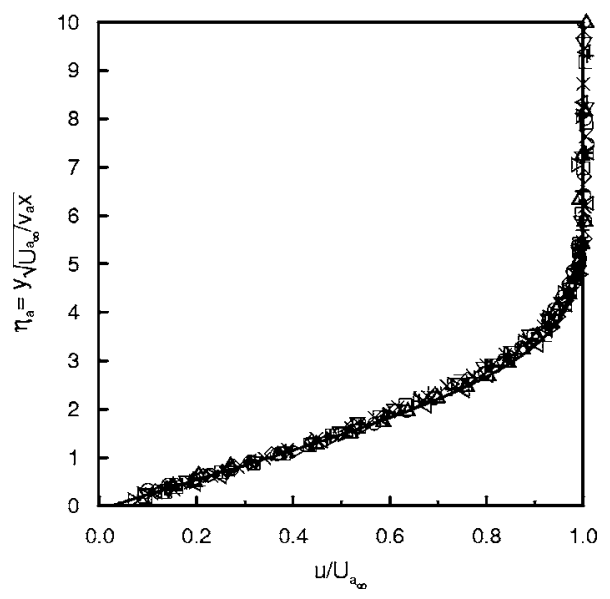

FIG. 2. Experimental and theoretical velocity profiles over the water surface. $\diamond, x=0.6 \mathrm{~m} ; \bigcirc, x=0.9 \mathrm{~m} ; \triangle, x=1.2 \mathrm{~m} ;+, x=1.55 \mathrm{~m} ; \times, x=1.9 \mathrm{~m}$; $\nabla, x=2.2 \mathrm{~m}$; and $\square, x=2.5 \mathrm{~m}$ for $U_{a_{\infty}}=3.77 \mathrm{~m} / \mathrm{s}$. $\triangleleft, x=0.6 \mathrm{~m}$, and $\triangleright, x$ $=2.5 \mathrm{~m}$ for $U_{a_{\infty}}=2.75 \mathrm{~m} / \mathrm{s},-$, Lock profile. $x$, distance from the leading edge. 


\section{Linear instability theory for two-layer flow}

To predict a preexisting sinusoidal wave superimposed on the interface in the flow system described in Sec. II B, it is assumed that the flow system is parallel, with the boundary layer of constant form in the streamwise direction. The wave amplitude $a$ is defined to be small compared with the wavelength, namely, steepness $k a \ll 1$. Also, $a$ is small in comparison with the thickness of the air boundary layer and therefore the change of the primary flow due to the surface disturbances can be ignored. A stream function based on normalmode solutions is introduced to describe the perturbed surface-induced motion in the form

$$
\psi(x, y, t)=\phi(y) e^{i k(x-c t)},
$$

where $k$ and $c$ are complex wave number and phase velocity, $k_{r}$ and $c_{r}$ represent the real parts, $k_{i}$ and $c_{i}$ represent the imaginary parts, $\sigma=k c$ is the angular frequency, and $\phi(y)$ is the amplitude function. Neglecting the product terms of wave-induced velocity and pressure disturbances, the Navier-Stokes equation is simplified to coupled OrrSommerfeld equations for the interfacial air and water layers, respectively. These take the forms

$$
\begin{aligned}
& \left(U_{a}-c\right)\left(\phi_{a}^{\prime \prime}-k^{2} \phi_{a}\right)-U_{a}^{\prime \prime} \phi_{a} \\
& \quad=-i\left(k \operatorname{Re}_{a \delta_{1 a}}\right)^{-1}\left(\phi_{a}^{\prime \prime \prime}-2 k^{2} \phi_{a}^{\prime \prime}+k^{4} \phi_{a}\right), \\
& \left(U_{w}-c\right)\left(\phi_{w}^{\prime \prime}-k^{2} \phi_{w}\right)-U_{w}^{\prime \prime} \phi_{w} \\
& \quad=-i\left(k \operatorname{Re}_{w \delta_{1 a}}\right)^{-1}\left(\phi_{w}^{\prime \prime \prime}-2 k^{2} \phi_{w}^{\prime \prime}+k^{4} \phi_{w}\right),
\end{aligned}
$$

where the primes denote the derivatives with respect to coordinate $y, y=y^{*} / \delta_{1}$, and Reynolds numbers are given as $\operatorname{Re}_{a \delta_{1 a}}=U a_{\infty} \delta_{1 a} / \nu_{a}, \operatorname{Re}_{w \delta_{1 a}}=U a_{\infty} \delta_{1 a} / \nu_{w}$. The free-stream velocity in the air, $U a_{\infty}$, and the boundary layer displacement thickness, $\delta_{1 a}$, were selected as the relevant velocity and length scales, respectively.

The boundary conditions at the interface are that the continuity of the velocity and shear stress and the discontinuity of the normal stress are balanced by the pressure and surface tension. Using the assumption of small wave amplitude, the boundary conditions are expanded at the mean water surface $y=0$ and higher-order terms are neglected. The result yields the four boundary conditions expressed in terms of $\phi$ as

$$
\begin{aligned}
& \phi_{a}=\phi_{w}, \\
& \frac{U_{a}^{\prime}}{c-U_{o}} \phi_{a}+\phi_{a}^{\prime}=\frac{U_{w}^{\prime}}{c-U_{o}} \phi_{w}+\phi_{w}^{\prime}, \\
& \frac{\mu_{a}}{\mu_{w}}\left\{\left(\frac{U_{a}^{\prime \prime}}{c-U_{o}}+k^{2}\right) \phi_{a}+\phi_{a}^{\prime \prime}\right\} \\
& =\left\{\left(\frac{U_{w}^{\prime \prime}}{c-U_{o}}+k^{2}\right) \phi_{w}+\phi_{w}^{\prime \prime}\right\},
\end{aligned}
$$

$$
\begin{aligned}
\frac{\rho_{a}}{\rho_{w}} & \left.\left(U_{a}^{\prime}-\frac{k c_{o}^{2}}{c-U_{o}}\right) \phi_{a}+\left(c-U_{o}+i \frac{3 k}{\operatorname{Re}_{a \delta_{1 a}}}\right) \phi_{a}^{\prime}-i \frac{\phi_{a}^{\prime \prime \prime}}{k \operatorname{Re}_{a \delta_{1 a}}}\right\} \\
= & \left\{\left(U_{w}^{\prime}-\frac{k c_{o}^{2}}{c-U_{o}}\right) \phi_{w}+\left(c-U_{o}+i \frac{3 k}{\operatorname{Re}_{w \delta_{1 a}}}\right) \phi_{w}^{\prime}\right. \\
& \left.-i \frac{\phi_{w}^{\prime \prime \prime}}{k \operatorname{Re}_{w \delta_{1 a}}}\right\},
\end{aligned}
$$

where

$$
\begin{aligned}
& c_{o}^{2}=(k \mathrm{Fr})^{-1}+k \mathrm{We}^{-1}, \\
& \mathrm{Fr}=\frac{U_{a_{\infty}}^{2}}{g \delta_{1 a}}, \\
& \mathrm{We}=\frac{U_{a_{\infty}}^{2} \delta_{1 a}\left(\rho_{w}-\rho_{a}\right)}{T} .
\end{aligned}
$$

The boundary conditions at infinity are

$$
\begin{aligned}
& y \rightarrow \infty: \phi_{a}=\phi_{a}^{\prime}=0, \\
& y \rightarrow-\infty: \phi_{w}=\phi_{w}^{\prime}=0 .
\end{aligned}
$$

The problem described above is to find an eigenvalue of the Orr-Sommerfeld equations. Only the spatial growth is considered in this study. Thus, a real angular frequency $\sigma_{r}$ is given and the eigenvalue $k$ is computed with the corresponding phase velocity $c$. The imaginary part of the complex wave number $k$ represents the growth rate. If $-k_{i}$ is positive, the wave grows. In contrast, if $-k_{i}$ is negative, the wave decays.

It is illuminating to calculate the amplification of the wave height as a function of downwind distance for direct comparison with the experimental measurements as a means of assessing the applicability and relevance of linear instability theory as the pertinent growth mechanism for gravitycapillary waves. The wave height at a distance $x$ downstream can be obtained by integrating the local growth rate, $-k_{i}$, as follows:

$$
\frac{H_{x}}{H_{o}}=\exp \int_{x_{o}}^{x}-k_{i}(x) d x,
$$

where $H_{o}$ is the initial wave height at position $x_{o}$. The variation in the local growth rate is caused by the growth of the boundary layer, which itself conflicts with the earlier assumption of parallel flow. This difficulty was assessed qualitatively in the experiments of Schubauer and Skramstad, ${ }^{14}$ who confirmed all the general characteristics predicted by linear instability theory over a fixed flat plate. This is because the growth of the boundary layer is slow in the flow direction and has a secondary effect on the wave growth rate through the change in velocity in the vertical direction. Nevertheless, the discrepancy arising from the actual nonparallel flow exists. ${ }^{29}$ As a first attempt to compare the spatial wave growth between the theory and experiment for a laminar two-phase flow model, the simplified formula (26) is adopted to calculate the wave height. 

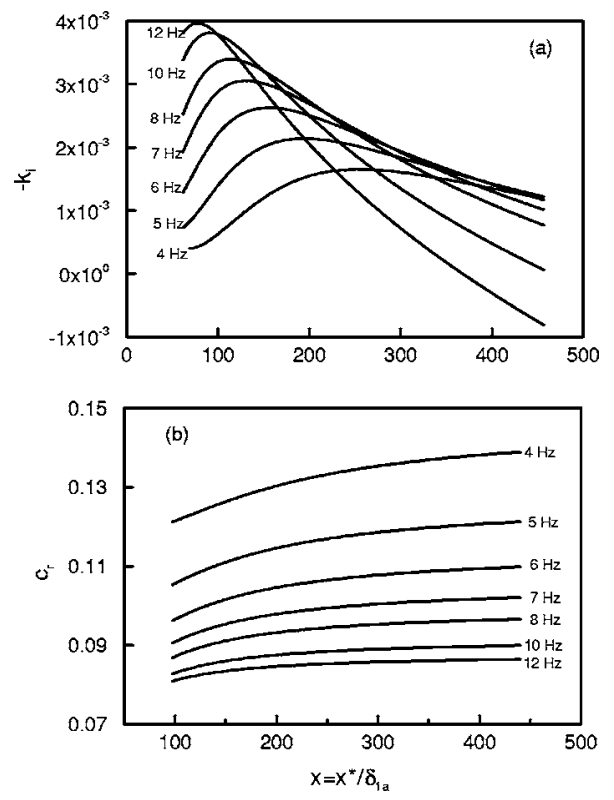

FIG. 3. Prediction of the spatial growth rate (a) and phase velocity (b) of water waves at different frequencies with $U_{a_{\infty}}=3.77 \mathrm{~m} / \mathrm{s}$.

\section{NUMERICAL PROCEDURES AND RESULTS}

\section{A. Numerical procedures}

The integration of the Orr-Sommerfeld equation was based on the fourth-order Runge-Kutta method through a conventional filter scheme to remove the so-called parasitic error. ${ }^{30} \mathrm{~A}$ code was written to conduct the integration. Digital values of the velocity profiles and their curvatures such as $U_{a}, U_{a}^{\prime \prime}, U_{w}$, and $U_{w}^{\prime \prime}$ were stored in a separate subroutine in different arrays with steps $\Delta y=0.01$ and were called by the main program during the computation. This started at $y$ $=5.5$ which is well above the outer edge of the air boundary layer (the air boundary layer thickness is $y=2.94$ for the Lock profile) and integrated downwards. To reduce execution time, the starting point in the water layer was at $y=-15$ and integration carried out upwards. Although this point was inside the water boundary layer, the errors in the eigenvalues were shown to be less than $0.2 \%$ from those when the calculation started at the point $y=-30$, approximately at the outer edge of the water boundary layer. When the two independent integrations met at the interface, a method of false position was employed to search the eigenvalue. The scheme has the advantage of being able to give a general estimation for the initial eigenvalue used for the iteration. $^{31}$

To assess the reliability of the present numerical model, the spatial instability of a Blasius flow on a rigid flat plate was examined in the absence of pressure gradient. A comparison with the simulation of Jordinson ${ }^{15}$ showed a good agreement between the two models. ${ }^{22}$

\section{B. Results using Lock profile}

Figure 3 shows the spatial growth rates and the corresponding phase velocities for different water wave frequencies calculated using the Lock profile with a free-stream ve- locity of $3.77 \mathrm{~m} / \mathrm{s}$. The main observation from this figure is that the growth rate changes with respect to the downwind distance due to the consistent development of a laminar boundary layer. This is different from the conventional approach which uses a log-linear profile to represent a fully developed turbulent flow and accordingly predicts a constant exponential growth rate. ${ }^{4,5,8}$ The present prediction reveals that for gravity-capillary water waves of a specific frequency, the growth rate initially increases and then decreases. For each curve there is a maximum value at the position where the waves are most unstable. At this point, the waves abstract energy from the wind most efficiently. The peak value shifts further downstream with waves of decreasing frequency. This indicates that a long wave can keep growing for a longer distance. In the region further downwind, the growth rate is seen to decay monotonically. The reason is that the energy dissipation caused by viscosity is larger than the energy obtained from the wind; finally, the growth rate falls to a negative value and the waves start to decay.

The values of the phase velocity increase in the initial stage and become constant at a large distance downwind. It is known from the study of wave-current interaction that a surface current increases the phase velocity of water waves when they travel in the same direction as the current. A stronger current gives rise to a larger phase velocity. ${ }^{32}$ In Lock's theory the surface flow induced by the upper laminar airflow is constant with respect to distance. The change in phase velocity reflects the variation of the shear effect arising from the growth of the boundary layer on both sides of the interface.

\section{Sensitivity to the shape of the velocity profiles}

It has been observed that the phase velocities measured in the present experiments are consistently smaller than the numerical simulations using the Lock profile (presented in Sec. V C). Since the phase velocity may be significantly affected by the wind-induced surface current, ${ }^{5}$ it is probable that the smaller phase velocities are caused by the surface current being smaller than $2.13 \%$ of the free-stream airflow as Lock predicted. To investigate the effect of a weaker surface current, new velocity profiles were created with values lying between the Lock profile and the Blasius profile. The boundary layer equations (8) and (9) were integrated with a range of different values for viscosity applied in the lower layer. The more viscous the fluid in the lower layer, the smaller the surface current generated by the wind. When the viscosity tended to infinity, there was no surface current, resulting in the Blasius profile, as is to be expected with such a simulated solid surface.

Figure 4 gives two examples of the variation in growth rates depending on the Blasius-type profiles for 5- and $12-\mathrm{Hz}$ waves acted on by a wind speed of $3.77 \mathrm{~m} / \mathrm{s}$. The different slip conditions are shown in the figure. The linear model shows that the growth rate is extremely sensitive to slight changes in the laminar profile. For example, for the $5-\mathrm{Hz}$ wave, the growth rate increases by a factor of approximately 2 for the $2 \%$ difference between Blasius and Lock profiles. This sensitivity in growth rate was reported by Van 

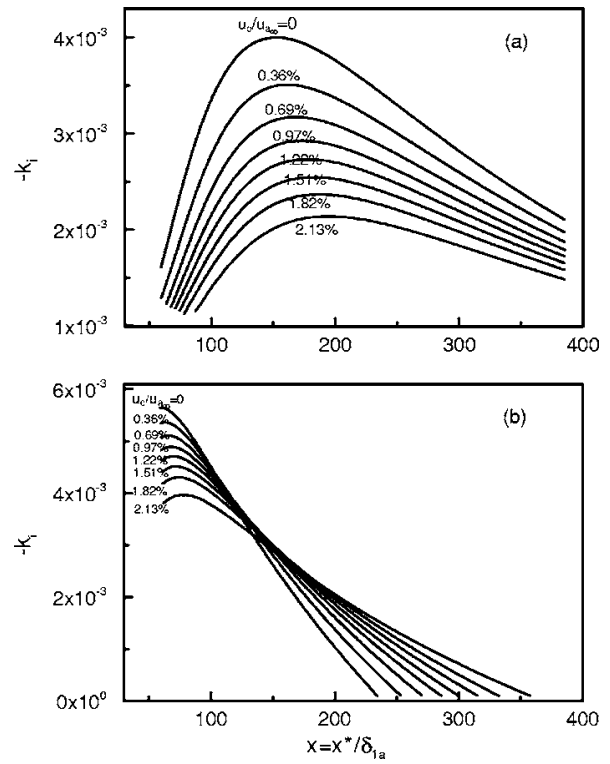

FIG. 4. The spatial growth rates for (a) $f_{0}=5 \mathrm{~Hz}$ and (b) $f_{0}=12 \mathrm{~Hz}$, calculated at a free-stream velocity $U_{a_{\infty}}=3.77 \mathrm{~m} / \mathrm{s}$ with different surface currents of $U_{o} / U_{a_{\infty}}$ shown in the figures. Surface current; $f_{0}$, perturbation frequency.

Gastel et al. ${ }^{5}$ and Morland and Saffman. ${ }^{33}$ For the $12-\mathrm{Hz}$ wave, the growth rate increases dramatically in the region $x<130$ and then greatly reduces for $x>130$. It appears that a reduction of the surface current and hence the increase of boundary layer thickness in the air give rise to a considerably larger growth rate. In contrast, the consequential modification of velocity profile in the water is the major effect causing the decrease of growth rate for high-frequency waves as they propagate further downstream. Accordingly, it is not only the velocity profile in the air but also the current profile in the water that are factors in transferring energy from wind to waves.

The corresponding phase velocities are shown in Fig. 5. The values systematically increase as the surface current increases. The results predicted by the linear instability theory demonstrate an agreement with the general wave-current theory, that is, when the surface current moves in the direction of wave propagation, the phase velocity is increased. Also, the calculations confirm the suggestion by Van Gastel et al. ${ }^{5}$ that the wind-induced surface current strongly affects the phase velocity.

Figure 6 presents an alternative way of describing the influence of the wind-generated surface current on the phase velocity. The curves were obtained using two free-stream velocities of 3.77 and $2.75 \mathrm{~m} / \mathrm{s}$. The minimum phase velocity for pure mechanical waves, $c_{m}$, is used as a nondimensional parameter. When subject to additional wind action, the modified phase velocities have the same trend as those for the pure mechanical water waves without wind action but the speeds are increased. This reveals the interesting phenomenon that the location of the minimum phase velocity shifts to a higher frequency and does not occur at a Bond number $B=1$. This is because the factor causing the minimum phase velocity is no longer related to the balance of gravity and surface-tension forces but also now involves the distribution
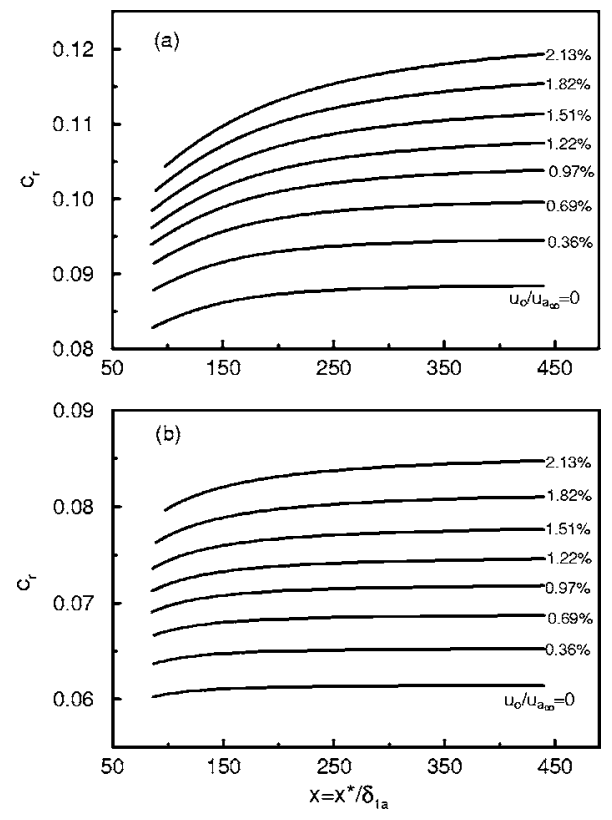

FIG. 5. Phase velocity distributions with respect to distance for (a) $f_{0}$ $=5 \mathrm{~Hz}$ and (b) $f_{0}=12 \mathrm{~Hz}$ with different surface currents of $U_{o} / U_{a_{\infty}}$.

of normal and shear stresses caused by the wind. A striking fact observed is that the value of the minimum phase velocity increases linearly with the surface current velocity.

\section{EXPERIMENTAL APPARATUS AND MEASUREMENT TECHNIQUES}

\section{A. Wind tunnel and wave tank}

The experiments were carried out in a low-speed and low turbulence wind tunnel with zero pressure gradient. Laminar airflow was maintained to at least a Reynolds number $\mathrm{Re}_{x}=6.8 \times 10^{5}$ on a flat rigid plate. Over a compliant water surface, the corresponding distance was sufficient to observe the initial stages in the development of gravitycapillary waves. A detailed description of the wind tunnel was given by $\mathrm{Tsai}^{22}$ and Tsai $e \mathrm{al.}^{23}$

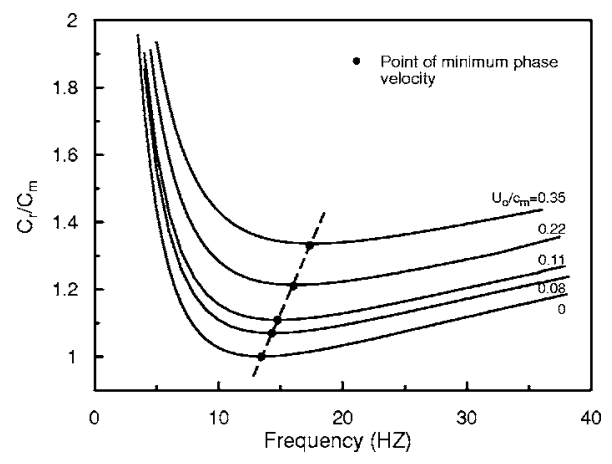

FIG. 6. Relationship of phase velocity to frequency with and without wind action. $c_{m}=0.232 \mathrm{~m} / \mathrm{s}$ is the minimum phase velocity for a pure mechanically generated water wave. Two groups of curves $\left(U_{o} / c_{m}=0.08,0.11\right.$ and $\left.U_{o} / c_{m}=0.22,0.35\right)$ were obtained using the wind speeds of 3.77 and $2.75 \mathrm{~m} / \mathrm{s}$ with the velocity profiles of $U_{o} / U_{a_{\infty}}=0.69 \%$ and $U_{o} / U_{a_{\infty}}$ $=2.13 \%$, respectively. The distance is $x=416$. 


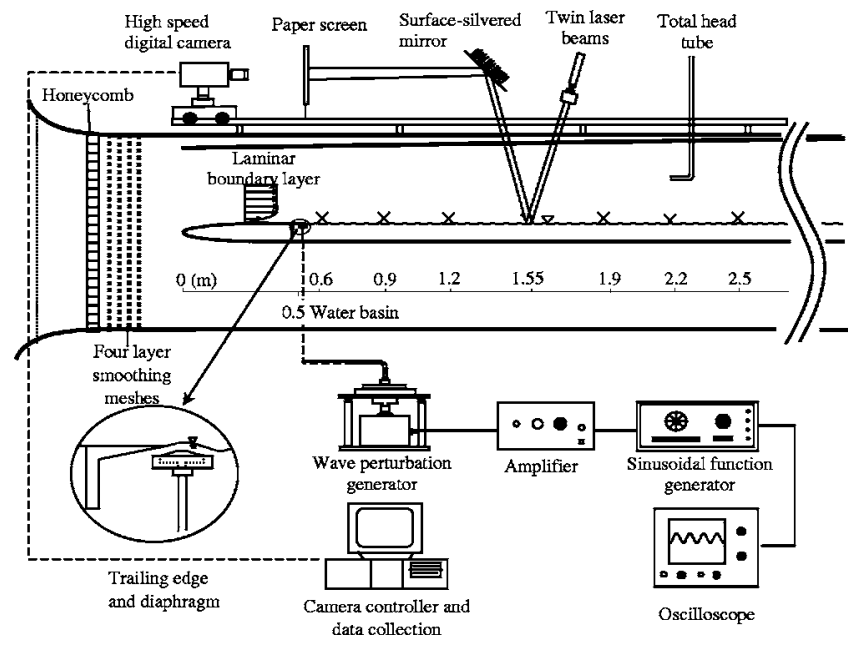

FIG. 7. Schematic of the wind tunnel layout and measurement technology. $\times$, the measurement stations.

A schematic representation of the experimental arrangement is shown in Fig. 7. A splitter plate with a profiled elliptical leading edge completely spanned the width of the tunnel at midheight. A water trough wave tank $4.5 \mathrm{~m}$ long $\times 71 \mathrm{~cm}$ wide $\times 5.5 \mathrm{~cm}$ deep was built into the plate. The depth was designed to produce gravity-capillary water waves in a deep-water condition. A rigid brass strip with a very sharp edge served as a trailing edge for the elliptical nose section, forming a solid/water interface to maintain continuity without disturbing the airflow at the upstream edge of the water basin. An absorbing beach $0.45 \mathrm{~m}$ in length was placed at the end of the tank to dissipate incoming water waves. No reflected waves were observed in the results. Since the smallscale water waves were extremely sensitive to any surface contamination, even a slightly contaminated water surface completely damped out high-frequency waves. For this reason, four drainage ports operated by an external tap were installed close to the water surface at the end of the tank in order to remove the surface film. This process was driven by fresh water supplied to the wave tank for 30 min before taking measurements, thereby keeping a clean environment.

\section{B. Generation and measurement of water waves}

Small-scale monochromatic gravity-capillary surface water waves were generated in the range of $5-12 \mathrm{~Hz}$. To agree with the theoretical linearized problem, the wave amplitude was required to be as small as possible. A novel technique was developed to give sensitive control over amplitude and frequency of these small waves using a pneumatically activated diaphragm (Fig. 7). The diaphragm was stuck over a brass strip with a recess of $8 \mathrm{~mm}$ wide $\times 2 \mathrm{~mm}$ deep $\times 68 \mathrm{~cm}$ long. A perturbation generator was responsible for providing periodic air pressure to the chamber enclosed by the diaphragm, and regular water waves were generated when the brass strip emerged into the water. A function generator produced a sinusoidal signal which was amplified before being connected to the driving arm. By adjusting the

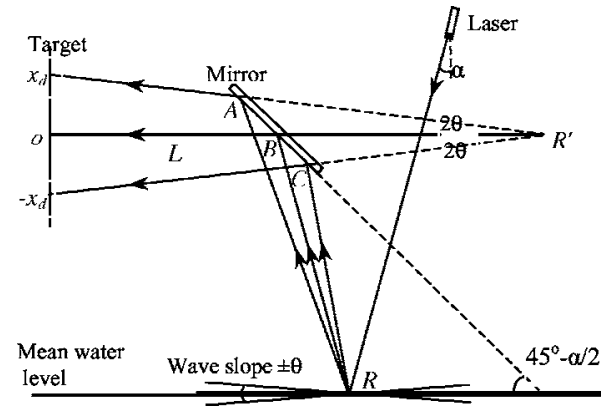

FIG. 8. Geometry of the laser beam traveling from the laser source to the target.

frequency in the function generator and the amplification, the wave frequency and amplitude could be set with the desired precision.

An optical technique was developed to measure the very small wave slopes from which the wave heights and phase velocities could be determined. This involved two parallel laser beams inclined at a small angle to the vertical, and a system of mirrors and screen. The phase difference between the continuous movement of the laser spots on the screen caused by the change of the surface wave slope yielded the necessary data. Figure 8 illustrates the path geometry of a laser beam from its source to the target screen. The wave surface slope $\theta$ is given by

$$
\theta=\frac{1}{2} \tan ^{-1} \frac{x_{d}}{L}
$$

where $x_{d}$ is the displacement of the laser spot from its original point with a still surface, and $L$ is the optical lever, which is the total traveling distance of the beam from the water surface to the target. In the experiment, the beams were set up at a small angle $\alpha=4^{\circ}$ to the vertical. The accuracy of this method depends on the length of the optical lever. A longer length gives a more accurate measurement of the wave slope because of the larger displacement of the spots on the screen. However, this is offset by the fact that the spots will move correspondingly faster. To ensure good resolution, a highspeed digital video camera was employed to photograph the spot movement.

\section{Laser source, high-speed digital camera and images}

The light source was a helium-neon laser, with minimum output power of $7 \mathrm{~mW}$ and beam diameter of $0.81 \mathrm{~mm}$. This diameter was much smaller than the shortest wave in the present study (19.4-mm wavelength for the $12-\mathrm{Hz}$ wave). The single beam was converted into two beams by an integral beam splitter and the beams were adjusted to be closely parallel using a rotating prism system. The spacing between the beams was $12 \mathrm{~mm}$ in the principal direction. A highspeed monochrome video camera was employed to capture the movement of the spots on the screen, operating at $500 \mathrm{frames} / \mathrm{s}$ in the experiments and recording over $4.2 \mathrm{~s}$ for a single data set. The locations of the two bright spots in the 
TABLE I. Initial conditions for the perturbed water waves with a fetch of $10 \mathrm{~cm}$.

\begin{tabular}{ccc}
\hline \hline $\begin{array}{c}\text { Frequency } \\
(\mathrm{Hz})\end{array}$ & $\begin{array}{c}H \\
(\mathrm{~mm})\end{array}$ & $k a$ \\
\hline 5 & 0.033 & $1.52 \times 10^{-3}$ \\
7 & 0.028 & $2.12 \times 10^{-3}$ \\
8 & 0.016 & $1.63 \times 10^{-3}$ \\
10 & 0.013 & $1.65 \times 10^{-3}$ \\
12 & 0.016 & $2.54 \times 10^{-3}$ \\
\hline \hline
\end{tabular}

image were recognized using image recognition software as two clusters. Their centroids were obtained using a light intensity weighted method. ${ }^{22}$

\section{Fourier analysis}

Fourier analysis was applied to separate the harmonic components of the wave slope time history when determining the wave phase velocity and height at the dominant frequency, that is, the diaphragm generated frequency $f_{0}$. The phase velocity calculated from the two laser beam measurements is

$$
c=\frac{2 \pi f_{0} \Delta s}{\Delta \phi},
$$

where $\Delta s$ and $\Delta \phi$ are the distance and the phase difference between the two laser beams, respectively. ${ }^{22}$ The surface elevation is the integration of the wave slope given by

$$
H=\frac{2 A \Delta s}{\Delta \phi},
$$

where $A$ is the slope amplitude of the dominant harmonic component. $^{22}$ The observed wave height $H$ and phase velocity $c$ are the most important directly measured wave parameters required for comparison with the theoretical predictions from linear instability theory.

\section{E. Accuracy of the measurements and system errors}

Water wave height and phase velocity were measured to an accuracy of $2 \%$, constrained by relative misalignment of the laser beams and the mirror. It should also be noted that there were unwanted waves in the water tank because the diaphragm generated not only long-crested waves traveling downstream but also corner waves and cross waves which caused some variation in the wave height with respect to distance. The error introduced by this effect was dependent on wave frequency, from approximately $20 \%$ root-meansquare (rms) wave height for $5 \mathrm{~Hz}$ down to $5 \%$ for $12 \mathrm{~Hz}$, and $5 \%$ error in phase velocity for all frequencies.

\section{EXPERIMENTAL RESULTS}

\section{A. Wave height}

The initial wave height and wave steepness observed at the first station along the flume are listed in Table I. These confirmed that extremely small waves could be generated. The development of the waves was examined under the ac-

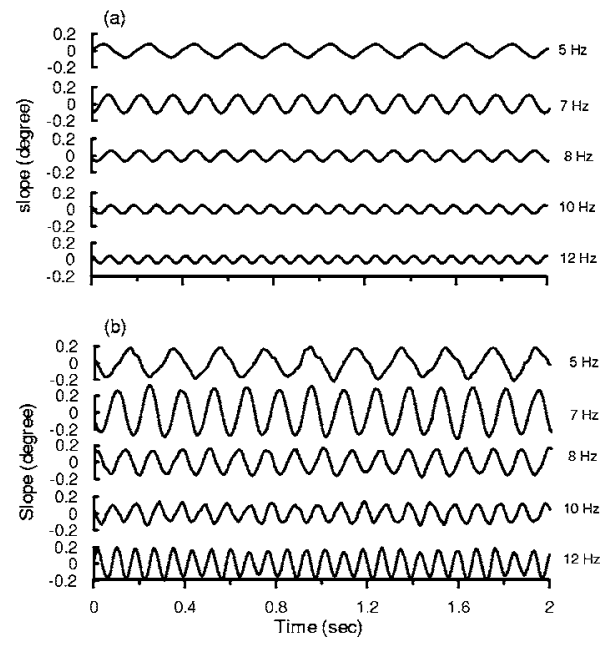

FIG. 9. Time history of wave slope measured at the fetch of $1.05 \mathrm{~m}$ (a) purely diaphragm perturbed water waves (b) acted on by a laminar air stream with the speed of $3.77 \mathrm{~m} / \mathrm{s}$.

tion of a wind speed of $3.77 \mathrm{~m} / \mathrm{s}$ which was equivalent to the velocity used in the numerical simulations. Five wave frequencies were tested. Figure 9 shows an example of the time history of wave slopes at a fetch of $1.05 \mathrm{~m}$. Under the action of the wind, the wave slope increases significantly but the waves still remain regular and two-dimensional.

The measurements of water waves were also carried out without wind action to understand the waves attenuation. Figure 10 shows the results. The theoretical curves predicted from (7) were fitted to the measurement points using a leastsquares procedure. In the present deep-water condition, wave damping was mainly contributed by the internal viscous effect. Sidewall friction had a secondary effect and bottom friction was negligible. For the $5-\mathrm{Hz}$ waves, there was a considerable scatter (approximately $20 \%$ rms) about the theoretical wave height curve. The variation is because the diaphragm wave generator not only produced two-dimensional waves propagating downstream, but also induced cross waves traveling in the spanwise direction and corner waves propagating at approximately $45^{\circ}$ to the longitudinal direction. The combination of these waves gives a complex variation of the wave height with a distance downwind. The 7and $8-\mathrm{Hz}$ waves show less scattering and good agreement with theory. A very good agreement is given by the two observations of the $10-$ and $12-\mathrm{Hz}$ waves, indicating minimal side effects from the wave tank for high-frequency waves.

In the case of wave damping for purely mechanical waves, the agreement between the experiment and theory demonstrates the very high accuracy of the present technique in measuring these very small waves. The wave height at $12 \mathrm{~Hz}$ was only $16 \mu \mathrm{m}$ at the first station and decayed to $2 \mu \mathrm{m}$ at the last station, $2 \mathrm{~m}$ downstream. A difference of $1 \mu \mathrm{m}$ could be detacted accurately. In addition, it was confirmed that the methods described above for removing the surface film were very effective since any surface contamination would have damped the wave amplitude rapidly. Visualization showed that, with illumination from above, the shadows of $12-\mathrm{Hz}$ waves were observed in a regular pattern on the bed to a fetch greater than $2 \mathrm{~m}$ with a clean water 

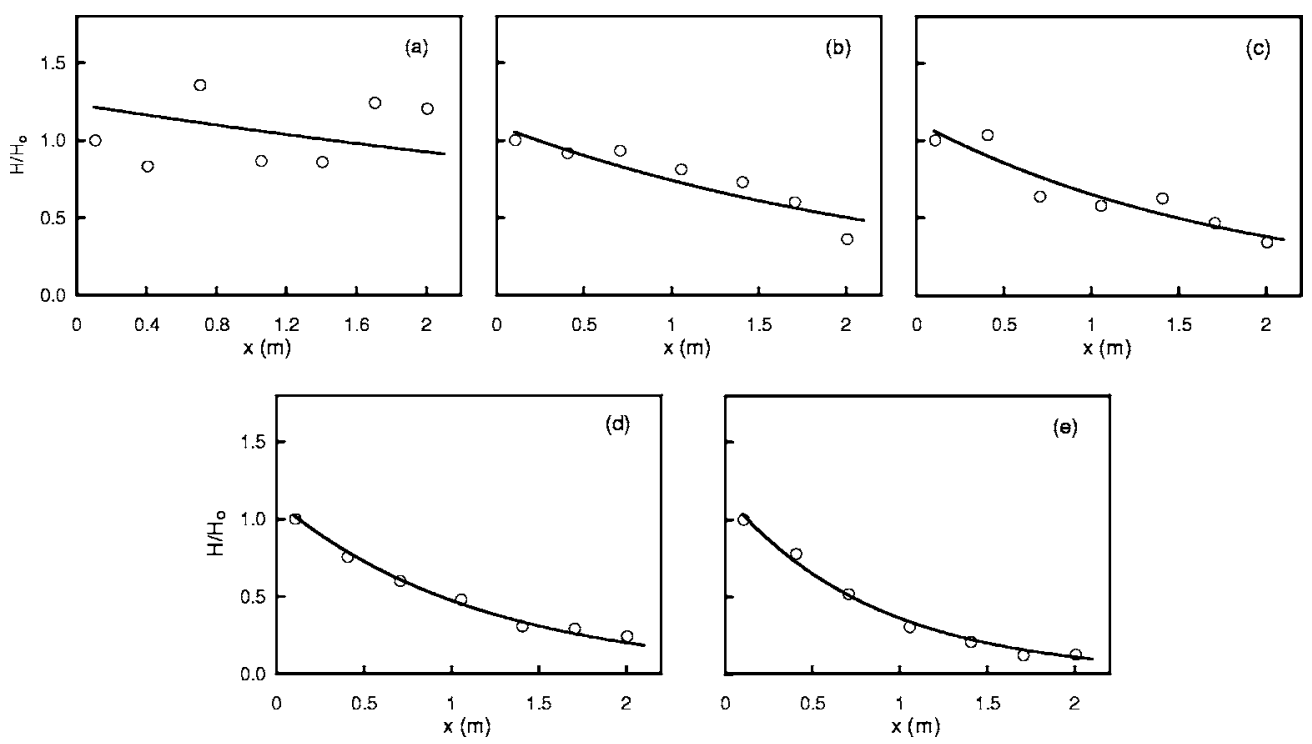

FIG. 10. Comparison of water wave decay between the theory and experiments; perturbation frequencies: (a) 5 , (b) 7, (c) 8 , (d) 10 , and (e) $12 \mathrm{~Hz}$. $H_{o}$, the wave height at the initial station.

surface. In contrast, the same waves completely disappeared within a fetch of $0.4 \mathrm{~m}$ when the water surface was slightly polluted (contaminated by stirring the water with a clean hand for several seconds). This technique was used to judge whether or not the water had a clean surface. Keeping the water extremely clean proved to be absolutely essential to the success of the present experiments. If a surfactant formed on the water surface, amplification of the wind waves would have been significantly reduced by the damping mechanism due to surface-tension gradients. ${ }^{34,35}$ This effect was not included in the present numerical model.

As discussed in Sec. III C, the spatial growth rate of water waves is highly sensitive to the form of the Blasiustype profile, which may be determined by the wind-induced surface current. Although in the present experiment the velocity distribution over the water surface has been measured very accurately, thereby demonstrating that a surface current has been generated by the airflow above, it is not appropriate to calculate the surface current by extrapolating the air velocity above the water surface. This is because the experimental error was approximately $4 \%$ in the near-wall region between 0.5 and $1 \mathrm{~mm}$ from the interface, which is larger than the discrepancy between the Blasius and Lock profiles. The only previous measurements reported in the literature of a surface flow sheared by a laminar airflow were conducted by Gupta $e t a l{ }^{24}$ They observed the surface current to be $3.5 \%$ of the free-stream velocity. This is significantly larger than the prediction of Lock. ${ }^{19}$ However, these experiments were conducted in the presence of a favorable pressure gradient. The greater shear stress on the surface as a consequence gave rise to a larger surface flow. In addition, the wave tank was short, 46 in. in length, and a strong and complex circulation was formed on the surface. Hence, the current was not purely induced by the upper shear airflow. Since there is no definite experimentally proven value for surface velocity under a laminar boundary layer and the present numerical model showed a better agreement with the observed phase velocities using $U_{o} / U_{a_{\infty}}=0.69 \%$, two theoretical lines corresponding to the velocity profile with surface currents $U_{o} / U_{a_{\infty}}=2.13 \%$ (the Lock profile) and $U_{o} / U_{a_{\infty}}=0.69 \%$ were tested to compare with the measured wave heights and phase velocities.

Figure 11 gives a comparison of the measured and theoretical wave heights for the wind speed of $3.77 \mathrm{~m} / \mathrm{s}$. The theoretical wave height was obtained by integration of the local growth rate presented in Fig. 3 using (26). Observations show that the maximum growth rate, corresponding to the most unstable wave, occurs in the present measurements for waves between 5 and $7 \mathrm{~Hz}$. A smaller amplification is found at 8 and $10 \mathrm{~Hz}$. At $12 \mathrm{~Hz}$, it was interesting to observe that the waves grow first and then decay at a large downwind distance. These growth (decay) trends for wave height are in reasonable agreement with the simulations. The two theoretical curves at 7 and $8 \mathrm{~Hz}$ have less discrepancy than at $5 \mathrm{~Hz}$, while the discrepancy becomes significant for the 10- and $12-\mathrm{Hz}$ waves. With a weaker surface current, $U_{o} / U_{a_{\infty}}$ $=0.69 \%$, shaping the velocity profile, the predictions show a faster decay of growth rate, particularly for the $12-\mathrm{Hz}$ wave. This is in agreement with the experimental data which show a distinct decay of wave height.

Both measurement and theory show that at a lower wind speed of $2.35 \mathrm{~m} / \mathrm{s}$, wave growth is smaller than that when acted on by the stronger wind $(3.77 \mathrm{~m} / \mathrm{s})$. For $10-\mathrm{Hz}$ waves, for example, the decay mechanism dominates the wave development and the wave height continually decreases. ${ }^{22}$

\section{B. Phase velocity}

The observations of the averaged phase velocities at all the measurement stations without the wind action are displayed in Fig. 12. The frequency range used in the present study gives an opportunity to test the well-known phenomenon of the minimum phase velocity using a Fourier analysis 

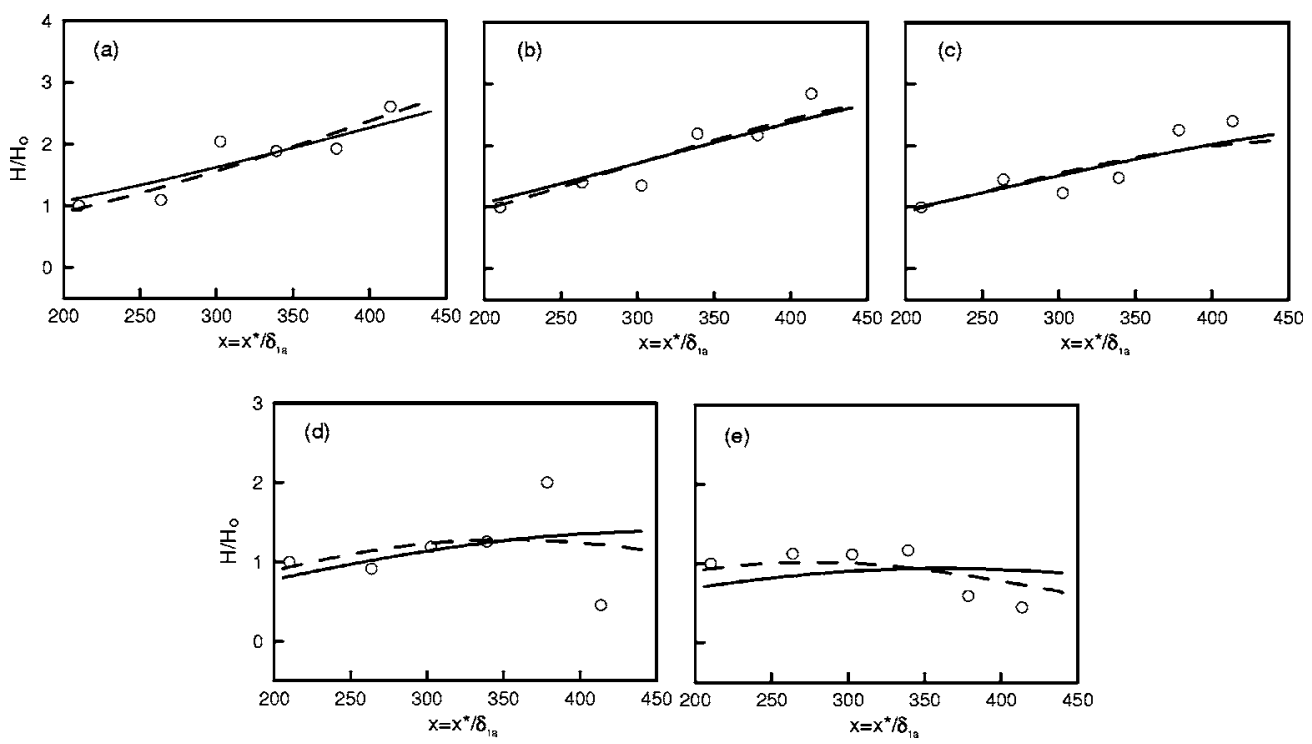

FIG. 11. Comparison of the experimental measurements and theoretical predictions of wave heights under the wind velocity of $3.77 \mathrm{~m} / \mathrm{s}$. Perturbation frequencies: (a) 5, (b) 7, (c) 8, (d) 10, and (e) $12 \mathrm{~Hz}$. Solid line, $U_{o} / U_{a_{\infty}}=2.13 \%$; dash line, $U_{o} / U_{a_{\infty}}=0.69 \%$.

to obtain higher harmonic components. The agreement proves that the surface-tension effect on short waves leads to the existence of a minimum phase velocity.

The influence of the wind action on phase velocity is displayed in Fig. 13. The comparison is made between the averaged phase velocities and the corresponding averaged theoretical values obtained at the same fetches as the measurement stations. Better agreement is obtained for the velocity profile with the weaker surface current of $U_{o} / U_{a_{\infty}}$ $=0.69 \%$. This may indicate that the surface current in the wave tank is smaller than that predicted by Lock. Van Gastel et $a l .{ }^{5}$ were able to show that the phase velocity was sensitive to the surface current but insensitive to the wind speed. Hence the surface current is likely to be the dominant factor in changing the phase velocity.

\section{DISCUSSION}

The results presented above show a reasonable agreement between the experiment and numerical predictions for the present study. Based on Miles' theory, ${ }^{1}$ Lighthill ${ }^{36}$ proposed a physical mechanism of energy transfer from wind to

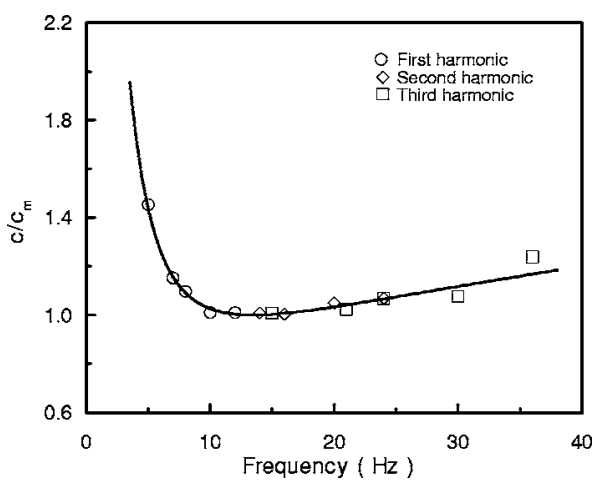

FIG. 12. Experimental phase velocities at the dominant and higher harmonic components in comparison with theoretical values. water waves in terms of a vortex force caused by the closed streamline around the critical layer. This is expressed by the formula

$$
E=\frac{\pi \rho_{a} c}{k}\left(\frac{U_{c}^{\prime \prime}}{U_{c}^{\prime}}\right) \overline{v_{c}^{\prime 2}}
$$

where the subscript $c$ denotes the critical level $y=y_{c}$ at which the airflow speed is equal to the surface wave phase velocity, that is, $U\left(y_{c}\right)=c$. The negative value of velocity curvature, the rate of change of vorticity, means that energy is abstracted from the wind and is transferred to the waves in the form of wave growth. This critical mechanism assumes that viscous effects can be ignored, and is believed to explain how a long gravity wave is amplified. For a wave of small phase velocity, Benjamin ${ }^{16}$ pointed out that the critical layer is too close to the boundary and hence the inviscid Reynolds stress formation in the critical layer is incapable of explaining the wave growth because the velocity profile near the boundary is linear and its curvature vanishes, particularly for a turbulent velocity profile with the critical point inside the

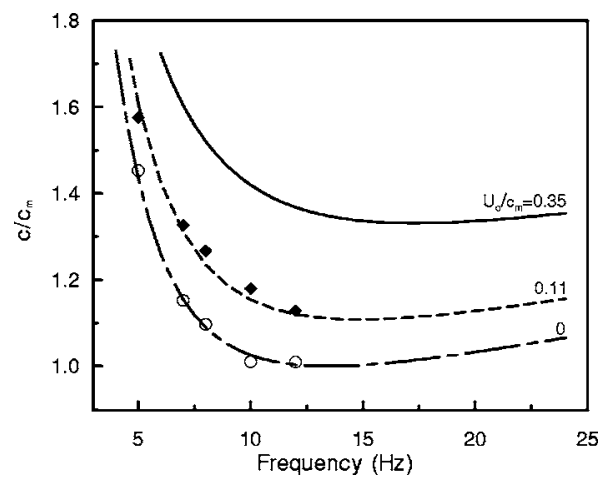

FIG. 13. Measurements and predictions of phase velocity at the wind speed of $3.75 \mathrm{~m} / \mathrm{s}$. Prediction: solid line, $U_{o} / U_{a_{\infty}}=2.13 \%$; dash line, surface current $U_{o} / U_{a_{\infty}}=0.69 \%$; solid-dash line $U_{o} / U_{a_{\infty}}=0$. 


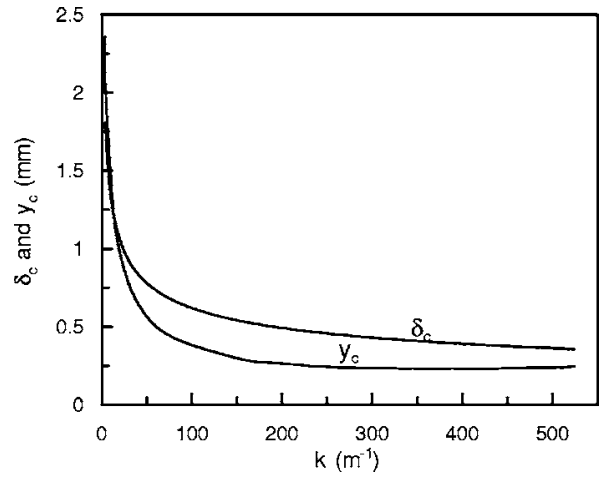

FIG. 14. Values of critical height $y_{c}$ and critical length $\delta_{c}$ over progressive waves with different wave numbers, calculated from Lock profile with $U_{a_{\infty}}=3.77 \mathrm{~m} / \mathrm{s}$.

laminar sublayer. For Blasius-type profiles, the value of curvature does not disappear. It can provide detailed information to verify if the critical mechanism explains the growth of short waves. In (30) the vertical velocity perturbed by the water waves is proportional to the wave slope. ${ }^{36}$ For a parallel flow and undisturbed velocity profile over a wave with the same frequency the discrepancy in $\overline{v_{c}^{\prime 2}}$ between the Blasius and Lock profiles is negligible. This point can be confirmed by assessing Eq. (22) from Lighthill. ${ }^{36}$ The only term to be considered is the ratio of the velocity curvature and gradient, $U_{c}^{\prime \prime} / U_{c}^{\prime}$. For a $5-\mathrm{Hz}$ wave with a phase velocity of $0.33 \mathrm{~m} / \mathrm{s}$, the longest water wave considered in the present study, at a fetch of $1 \mathrm{~m}$ and with $U_{a_{\infty}}=3.77 \mathrm{~m} / \mathrm{s}$, the critical height is $0.53 \mathrm{~mm}$ for the Blasius profile and $0.39 \mathrm{~mm}$ for the Lock profile. The ratio of $\left(U_{c}^{\prime \prime} / U_{c}^{\prime}\right)_{\text {Blasius }}$ to $\left(U_{c}^{\prime \prime} / U_{c}^{\prime}\right)_{\text {Lock }}$ is 1.07. In other words, the energy-transfer rate from the Blasius profile is only $7 \%$ higher than that from the Lock profile. However, the calculation shown in Fig. 4 demonstrates that the ratio of growth rates between the two profiles is approximately an order of two. From the discrepancy between inviscid theory and the full solution of the Orr-Sommerfeld equations it can be concluded that the critical mechanism is unable to explain the amplification of small waves even without the curvature reducing to zero in the critical layer.

Benjamin $^{2}$ suggested that viscosity is likely to have a significant effect on the wave growth through a thin wall friction layer only if the critical height $y_{c}$ is of the same order as $\delta_{c}$, where $\delta_{c}=\left(\nu_{a} / U_{c}^{\prime} k\right)^{1 / 3}$ denotes a characteristic length for the critical layer. Figure 14 gives the values of $y_{c}$ and $\delta_{c}$ using the Lock profile for the wind speed of $3.77 \mathrm{~m} / \mathrm{s}$ at different wave numbers. For waves with frequency greater than $1 \mathrm{~Hz}$, the two values are of the same order. Thus, viscosity appears to play an important role for free-surface wave instability, similar to that of serving as an agent to destabilize the Tollmien-Schlichting wave on a rigid plate in a single-phase flow. In Benjamin's theory, the viscosity in the wall frictional layer produces a nonuniform shear stress distributed on the wavy boundary, having a maximum value at the location ahead of the top of a wave and hence significantly influences the phase of the pressure in relation to the surface elevation. As a result, the increase of the pressure at the leeward side generates net drag on the free surface. This sheltering model of laminar flow over a hump could apply also to the mechanism of amplification for wind over water waves. $^{11,37}$

Miles ${ }^{3}$ proposed that energy is transferred from the airflow to waves through the viscous Reynolds stress in the region close to the water surface. He suggested that resonance between the Tollmien-Schlichting waves and freesurface waves is the mechanism responsible for the development of short water waves. A wind-induced growth rate, $\zeta_{a}$, was derived by solving the Orr-Sommerfeld equation, generalizing the work by Miles ${ }^{1}$ and Benjamin. ${ }^{2}$ The given formula was based on a turbulent log-linear profile, but this is not applicable to the present Lock profile and cannot be compared with the present study. Nevertheless, Miles ${ }^{3}$ illustrated that the net growth rate of water waves, $\zeta_{e}$, is the combination of the work done by the airflow and a negative viscous dissipation $\zeta_{\nu}=-2 \nu_{w} k^{2}$ for a deep-water wave. The net growth rate in space is

$$
\zeta_{e}=-k_{i}=\zeta_{a}+\zeta_{\nu}
$$

A water wave with a specific frequency becomes unstable only when the viscous dissipation in the water is not sufficient to balance the energy transferring to it from the wind. The analytical solution of Benjamin ${ }^{2,38}$ and Van Gastel et al. ${ }^{5}$ shows that pressure is the dominant force responsible for transferring energy from wind to waves through the component in phase with the wave slope. The work is done by the term $c p_{s} \partial \eta / \partial x$, where $p_{s}$ is the pressure on the surface and $\eta$ is the surface elevation. From this point of view, a high-frequency wave with higher steepness can extract energy from the wind more efficiently, resulting in a higher wind-induced growth rate, $\zeta_{a}$. Nevertheless, the viscous damping agent, $\zeta_{\nu}$, is also enhanced. The net growth rate for a high-frequency wave can only be positive for a short distance. When the damping mechanism becomes dominant as the wave propagates further downstream, the wave height decays even in the presence of wind action. This explains the theoretical curves and experimental results in Fig. 11 for 10and $12-\mathrm{Hz}$ waves. In contrast, low-frequency waves with smaller steepness abstract energy more slowly while the viscous damping is much smaller. The waves keep growing for a longer distance in Fig. 11 for 5- and 7-Hz waves. The balance between the wind-induced growth rate and the viscous damping rate can also explain the spatial growth rate curves in Fig. 3; that is, why the maximum value of the growth rate decreases and occurs at greater downwind distances as the wave frequency decreases.

The generation of the surface water current can modify the Tollmien-Schlichting waves in the airflow. According to Miles' resonance theory, the modified Tollmien-Schlichting waves will certainly change the growth of water waves. This may explain why the growth rate is sensitive to the Blasiustype profile. Physically, a movable boundary layer can reduce the drag significantly, and the momentum flux from air to water is decreased accordingly. This is why the Blasius profile without a surface current gives a significantly larger growth rate for the compliant surface than the Lock profile. However, further study is required to verify if the mechanism 
can increase the growth rate by a factor of 2 due to $2 \%$ discrepancy of the velocity profile.

Phase velocities were in better agreement with the simulations using $U_{o} / U_{a_{\infty}}=0.69 \%$ indicating that the windinduced current in the wave tank may be lower than Lock's prediction of $2.13 \%$ of the free-stream velocity. In Lock's theory, the water is infinite, which is different from the present wind tunnel experiments with a limited water tank. Hence, sidewall friction and water setup caused by wind shear may reduce the effective surface flow. Nelson et al. ${ }^{39}$ formulated a nonsimilar analytic solution for laminar airflow shearing over a thin water layer on a flat plate. The solution showed that the height of the surface film was proportional to $x^{1 / 4}$ and the surface current calculated using their method is only about $0.14 \%$ of the free-stream velocity. The present experiment with a deeper water layer is different from the shallow water film study presented by Nelson et al. However, their study suggests the possibility of a smaller surface current than Lock's prediction.

Regarding the present linear model, it is based on the assumption of parallel flow. In other words, the boundary layer is assumed to be uniform with respect to distance. This is counter to the real experimental situation. Barry and Ross $^{29}$ introduced a nonparallel flow theory in their investigation of the stability of the Blasius profile on a flat plate. The numerical simulation reported a reduction of the critical Reynolds number from 520 to 500, giving a better agreement with the measurements of Schubauer and Skramstad ${ }^{14}$ and Ross et al. ${ }^{40}$ Although the nonparallel flow may be a secondary effect, it is interesting to note the sensitive influence of the nonparallel theory applied in the present coupled airwater two-phase flow system.

\section{CONCLUSIONS}

A numerical simulation and delicate experiments have been conducted to test linear instability theory for a laminar air/water coupled flow system. Using the Lock profile, which was confirmed in the present measurements, the computation shows that the spatial wave growth rate varies in the wind direction depending on the development of the boundary layer and the frequency of externally generated disturbances. In particular, the numerical model shows that the growth rate is extremely sensitive to the form of the laminar Blasius-type profile modified by the water surface flow. The prediction is supported by measurements of wave height, especially using the velocity profile with $U_{o} / U_{a_{\infty}}=0.69 \%$ (for a wind speed of $3.77 \mathrm{~m} / \mathrm{s}$ ). Measurement of the phase velocities for incident waves and their harmonics confirms the well-known theoretical curve showing the effect of surface tension on the variation in wave phase speed with wavelength. Under the action of an additional wind stress, the minimum phase velocity increases; this is also supported by the results from the present experiments.

Agreement between the measurements and theory suggests that the growth (decay) process of small-scale waves at the initial stage sheared by a laminar airflow can be explained by linear instability theory. The results support the original Benjamin-Miles idea of a linear model including the effect of viscosity for the small waves, when the profiles used in the works of Benjamin ${ }^{2}$ and Miles ${ }^{3}$ are replaced by the Lock profile.

\section{ACKNOWLEDGMENTS}

The authors wish to express their thanks to Professor J. C. R. Hunt for his helpful comments on the manuscript during preparation. We are most grateful to Professor F. T. Smith for discussions on the numerical model and Professor J. Z. Yim for reviewing of this paper. We are also indebted to Image Diagnostics Ltd. for a free extended loan of the camera system, and Keith Harvey, Bill Fairman, and Paul Mennel for their technical support in construction of the wind tunnel and operation of the experimental equipment.

${ }^{1}$ J. W. Miles, "On the generation of surface waves by shear flows," J. Fluid Mech. 3, 185 (1957).

${ }^{2}$ T. B. Benjamin, "Shearing flow over a wavy boundary," J. Fluid Mech. 6, 161 (1959).

${ }^{3}$ J. W. Miles, "On the generation of surface waves by shear flows. Part. 4," J. Fluid Mech. 13, 433 (1962).

${ }^{4} \mathrm{G}$. R. Valenzuela, "The growth of gravity-capillary waves in a coupled shear flow," J. Fluid Mech. 76, 229 (1976).

${ }^{5}$ K. Van Gastel, P. A. E. M. Janssen, and G. J. Komen, "On phase velocity and growth rate of wind-induced gravity-capillary waves," J. Fluid Mech. 161, 199 (1985).

${ }^{6} \mathrm{G}$. H. Wheless and G. T. Csanady, "Instability waves on the air-sea interface," J. Fluid Mech. 248, 363 (1993).

${ }^{7}$ T. R. Larson and J. W. Wright, "Wind-generated gravity-capillary waves: Laboratory measurements of temporal growth rates using microwave backscatter," J. Fluid Mech. 70, 417 (1975).

${ }^{8}$ S. Kawai, "Generation of initial wavelets by instability of a coupled shear flow and their evolution to wind waves," J. Fluid Mech. 93, 661 (1979).

${ }^{9} \mathrm{H}$. Kawamura and Y. Toba, "Ordered motion in the turbulent boundary layer over wind waves," J. Fluid Mech. 197, 105 (1988).

${ }^{10}$ M. Fulgosi, D. Lakehal, S. Banerjee, and V. De Angelis, "Direct numerical simulation of turbulence in a sheared air-water flow with a deformable interface," J. Fluid Mech. 482, 319 (2003).

${ }^{11}$ A. J. Grass, R. J. Stuart, and M. Mansourtehrani, "Vortical structures and coherent motion in turbulent flow over smooth and rough boundaries," Philos. Trans. R. Soc. London, Ser. A 336, 35 (1991).

${ }^{12}$ S. E. Belcher and J. C. R. Hunt "Turbulent flow over hills and waves," Annu. Rev. Fluid Mech. 30, 507 (1998).

${ }^{13}$ H. Schlichting, Boundary Layer Theory, 7th ed. (McGraw-Hill, New York, 1979).

${ }^{14}$ G. B. Schubauer and H. K. Skramstad, "Laminar boundary-layer oscillations and stability of laminar flow," J. Aeronaut. Sci., 14, 69 (1947).

${ }^{15}$ R. Jordinson, "The plate boundary layer. Part 1 . Numerical integration of the Orr-Sommerfeld equation," J. Fluid Mech. 43, 801 (1970).

${ }^{16}$ T. B. Benjamin, "Fluid flow with flexible boundaries," general lecture in 11th International Congress on Theoretical and Applied Mechanics, Munich, Germany, 30 August-5 September 1964 (Springer, Berlin, 1964).

${ }^{17}$ P. J. Blennerhassett, "On the generation of waves by wind," Philos. Trans. R. Soc. London, Ser. A 298, 451 (1980).

${ }^{18}$ P. J. Blennerhassett and F. T. Smith, "Short-scale waves on wind-driven water ('cat's paws')," Proc. R. Soc. London, Ser. A 410, 1 (1987).

${ }^{19}$ R. C. Lock, "The velocity distribution in the laminar boundary layer between parallel streams," Q. J. Mech. Appl. Math. 4, 42 (1951).

${ }^{20} \mathrm{R}$. C. Lock, "Hydrodynamic stability of the flow in the laminar boundary layer between parallel streams," Proc. Cambridge Philos. Soc. 50, 105 (1954).

${ }^{21}$ T. R. Akylas, "A nonlinear theory for the generation of water waves by wind,” Stud. Appl. Math. 67, 1 (1982).

${ }^{22}$ Y. S. Tsai, "The interaction of gravity-capillary water waves with a laminar air flow," Ph.D. thesis, University of London, 2002.

${ }^{23}$ Y. S. Tsai, A. J. Grass, and R. R. Simons, "Experimental study of a laminar air boundary layer over a water surface," Exp. Fluids 35, 472 (2003).

${ }^{24}$ A. K. Gupta, M. T. Landahl, and E. L. Mollo-Christensen, "Experimental and theoretical investigation of the stability of air flow over a water surface," J. Fluid Mech. 33, 673 (1968). 
${ }^{25}$ H. Lamb, Hydrodynamics (Cambridge University Press, Cambridge, (1932).

${ }^{26} \mathrm{~J}$. N. Hunt, "Viscous damping of waves over an inclined bed in a channel of finite width," Houille Blanche 7, 836 (1952).

${ }^{27}$ M. J. Lighthill, Waves in Fluids (Cambridge University Press, Cambridge, (1978).

${ }^{28} \mathrm{M}$. Gaster, "A note on the relation between temporally-increasing and spatially-increasing disturbances in hydrodynamic stability," J. Fluid Mech. 14, 222 (1962).

${ }^{29}$ M. D. J. Barry and M. A. S. Ross, "The flat plate boundary layer. Part 2. The effect of increasing thickness on stability," J. Fluid Mech. 43, 813 (1970).

${ }^{30}$ R. E. Kaplan, "The stability of laminar incompressible boundary layers in the presence of compliant boundaries," Massachusetts Institute of Technology Aeroelastic and Structures Research Laboratory Report No. ASRL-TR 116-1, 1964.

${ }^{31}$ P. W. Carpenter and A. D. Garrad, "The hydrodynamic stability of flow over Kramer-type compliant surface. Part 1. Tollmien-Schlichting instabilities,” J. Fluid Mech. 155, 465 (1985).
${ }^{32}$ I. G. Jonsson, The Sea: Ocean Engineering Science (Wiley, New York, 1990), Vol. 9, pp. 65-120.

${ }^{33}$ L. C. Morland and P. G. Saffman, "Effect of wind profile on the instability of wind blowing over water," J. Fluid Mech. 252, 383 (1993).

${ }^{34}$ D. B. Creamer and J. A. Wright, "Surface films and wind wave growth," J. Geophys. Res. 97, 5221 (1992).

${ }^{35}$ O. Saetra, "Effects of surface film on the linear stability of an air-sea interface," J. Fluid Mech. 357, 59 (1998).

${ }^{36}$ M. J. Lighthill, "Physical interpretation of the mathematical theory of wave generation by wind," J. Fluid Mech. 14, 385 (1962).

${ }^{37}$ J. C. R. Hunt, “Thomas Brooke Benjamin,” Biogr. Mem. Fellows R. Soc. 49, 39 (2003).

${ }^{38}$ T. B. Benjamin, "The threefold classification of unstable disturbances in flexible surfaces bounding inviscid flows," J. Fluid Mech. 16, 436 (1963).

${ }^{39}$ J. J. Nelson, A. E. Alving, and D. D. Joseph, "Boundary layer flow of air over water on a flat plate" J. Fluid Mech. 284, 159 (1995).

${ }^{40}$ J. A. Ross, F. H. Barnes, J. G. Burns, and M. A. S. Ross, "The flat boundary layer. Part 3. Comparison of theory with experiment," J. Fluid Mech. 43, 819 (1970) 